\title{
Therapeutic Options in the Management of Malignant Glaucoma
}

\author{
Robert J Campbell, Mark Fava, Sherif R El-Defrawy \\ Queen's University, Department of Ophthalmology, Hotel Dieu Hospital, Kingston Ontario, Canada
}

\section{INTRODUCTION}

Malignant glaucoma (MG) is a potentially sight-threatening entity. As understanding of the pathophysiologic events underlying this disorder has expanded, a wide variety of treatment modalities have been described and are reviewed herein. The term malignant glaucoma was coined by von Graefe in 1869 to describe a clinical syndrome of axial anterior chamber shallowing and higher than expected intraocular pressure despite the presence of a patent peripheral iridectomy. The term was chosen to reflect the generally poor outcome of this condition and its refractoriness to therapy. Since that time a number of alternative names have been suggested in an attempt to provide a name descriptive of the pathophysiology. While many of these names provide accurate descriptions of some of the events involved in this disease process, our incomplete understanding of all the events involved in the pathophysiology of MG makes it difficult to develop a universally accepted name for this disorder. Moreover, while precision of language is an important goal, the lack of universal usage of some alternative names limits their utility for the purposes of clinical discussion. Thus, while clearly imperfect, the term malignant glaucoma is widely used, makes no assumptions regarding pathophysiology and is understood to refer to a specific syndrome with defined clinical findings. Thus, we have employed this term in this review.

\section{PATHOPHYSIOLOGY}

Although the pathophysiologic events underlying malignant glaucoma are not completely and unequivocally understood, some aspects are widely accepted. Shaffer proposed that in MG aqueous enters and is retained within or behind the vitreous body. ${ }^{1} \mathrm{~A}$ relative block to the forward movement of aqueous from the vitreous cavity into the posterior and anterior chambers also plays an important role in the sequestration of aqueous within the vitreous cavity. ${ }^{2}$ This block is felt to occur mainly at the anterior vitreous face, ciliary processes and lens equator. Anatomically, the surface area available for anterior diffusion of aqueous across the anterior vitreous decreases significantly with anterior movement of the vitreous body. ${ }^{3}$ Additionally, pressurization and compression of the vitreous decreases its permeability to aqueous. ${ }^{4}$ Thus, the accumulation of fluid in the posterior portions of the eye exacerbates the relative block to forward movement of fluid and a vicious cycle ensues with increasing posterior pressure, forward movement of the irislens diaphragm, and axial shallowing of the anterior chamber (Figs 1 and 2).

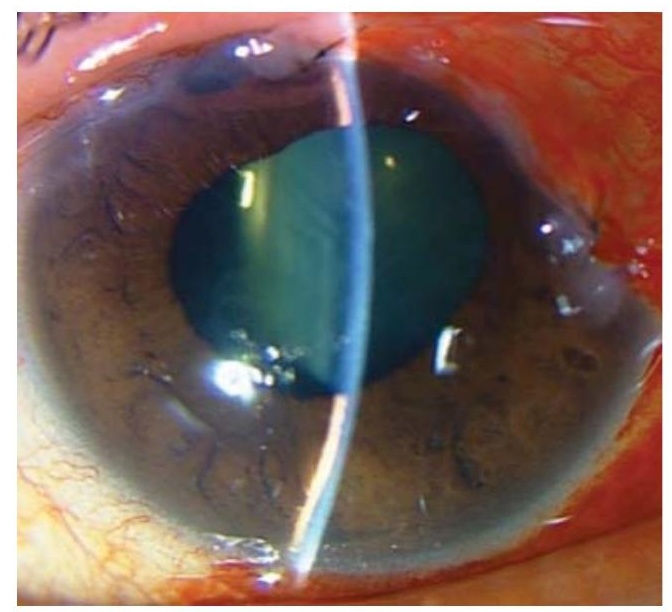

Fig. 1: Clinical photograph of an eye with malignant glaucoma. Note axial shallowing of the anterior chamber. Intraocular pressure was $42 \mathrm{~mm} \mathrm{Hg}$

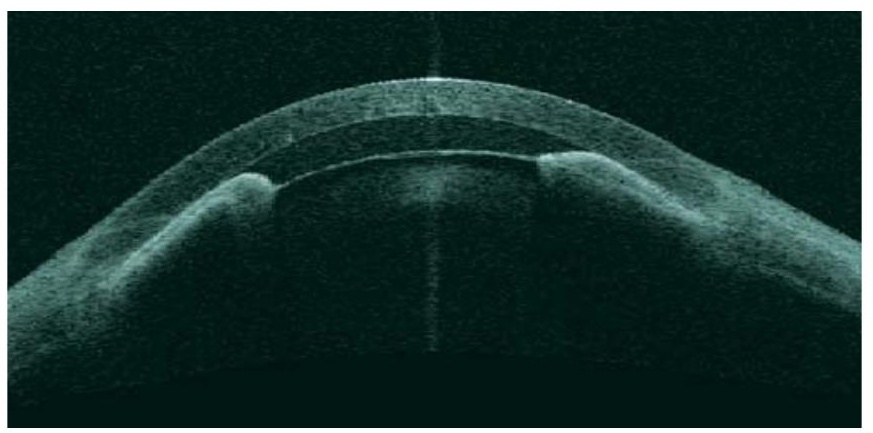

Fig. 2: Anterior segment optical coherence tomography of an eye with malignant glaucoma. Courtesy. Dr. Tanuj Dada 
A number of possible initiating events have been implicated in the pathogenesis of MG. Surgically-induced egress of fluid from the anterior chamber with associated forward movement of the iris-lens diaphragm may set up the necessary ciliary bodyvitreous configuration for the development of MG. ${ }^{5-7}$ Alternatively, choroidal expansion may push the iris-lens diaphragm forward with similar results. ${ }^{3}$ Regardless of the initial event, once the vitreous moves forward against the ciliary processes, aqueous may be directed into or behind the vitreous body. This leads to further compression of the vitreous and a reduction in the anterior hyaloid surface area available for aqueous diffusion, thereby establishing the circumstances for MG.

\section{PREVENTION}

As with many surgical complications and disease processes, prevention is an important route to minimizing the morbidity associated with malignant glaucoma. Recognizing the risk factors for this condition may alert the physician to take precautionary actions. The primary risk factors include short axial length and hyperopia, with their associated crowded anterior segment and chronic angle closure, as well as zonular laxity. ${ }^{3,8}$ The latter risk factor may be associated with pseudoexfoliation syndrome. $^{5}$

When undertaking intraocular surgery, and in particular glaucoma surgery, in patients at elevated risk for MG, a number of technical adjustments may decrease the likelihood of developing this complication. Fastidious attention to the maintenance of anterior chamber depth both intra and post operatively helps prevent anterior movement of the vitreous body, which plays a role in the initiation of MG in some cases. ${ }^{5,9,10}$ Furthermore, avoidance of hypotony helps prevent choroidal expansion or frank effusion, which may also play a role in initiating MG through the induced anterior shift of the vitreous body. ${ }^{3}$ To achieve these goals, relatively tight wound closure and judicious suture release in patients undergoing trabeculectomy are important. In eyes at high-risk of MG, the surgeon should also consider anterior chamber reformation at an earlier stage should shallowing secondary to overfiltration occur following glaucoma surgery. This pre-emptive intervention may interrupt the cascade of events leading to MG. Discontinuation of cycloplegics should be undertaken with care in any patient considered at high-risk of MG. ${ }^{5,10,11}$ Finally, prophylactic vitrectomy has been described for high risk eyes undergoing phacoemulsification. ${ }^{12}$ Although the results of this small study were positive, when considering this prophylactic surgical intervention, one should consider that MG is a rare event and vitrectomy is not without risk.

\section{MEDICAL THERAPIES}

As our understanding of the pathophysiology involved in MG has evolved, so too have the approaches to medical therapy of this disorder. Currently accepted medical treatments are directed at restoring physiologic configurations of the ciliary body, vitreous and lens, and the re-establishment of a more normal posterior chamber through which aqueous may freely flow from the ciliary body to the anterior chamber. To this end, as first described by Chandler, cycloplegics and mydriatics are the primary therapies. ${ }^{13}$ Atropine $1 \%$ two to four times daily in combination with phenylephrine $10 \%$ four times daily is a commonly described regimen.

Aqueous suppressants and hyperosmotics have also been used successfully in the treatment of MG. ${ }^{2,10,14}$ These agents decrease the rate of aqueous infusion into the vitreous and also deturgesce the vitreous and choroid. This latter effect may enhance the ability of cycloplegia to return the ciliary body, lens and vitreous back to physiologic locations. ${ }^{3}$ Thus, numerous reports suggest that the combination of cycloplegia, aqueous suppressant and hyperosmotic therapies is an appropriate and rational approach.

Finally, steroids have been widely suggested to be helpful in the treatment of MG. ${ }^{10,14}$ These drugs-usually delivered topically but occasionally systemically-may provide therapeutic benefit in multiple ways. By decreasing blood vessel permeability, steroids may decrease the tendency for choroidal expansion or ciliary body swelling and rotation. Steroids may also improve vitreous permeability as inflammation may negatively impact the fluid conductivity of the vitreous gel. ${ }^{15}$ Finally steroids may decrease the rate of peripheral anterior synechiae formation thereby improving the long-term prognosis for IOP control. Overall, medical therapy has been found to successfully treat MG in approximately $50 \%$ of cases, with resolution often requiring up to five days of treatment. ${ }^{5,10,16}$

\section{SURGICAL THERAPIES}

If medical therapies fail to resolve MG, surgical interventions should be considered. The accepted initial steps have evolved over time as new understanding and technologies have arrived.

Mechanistically, current surgical approaches attack the problem in two primary ways. First, they may create a new communication through the anterior vitreous to allow fluid to exit from regions of sequestration within or behind the vitreous gel (Fig. 3). Second, as in medical treatments, surgical interventions may attempt to restore physiologic configurations of the ciliary body, vitreous and lens, and thereby re-establish a posterior chamber through which aqueous may flow in a physiologic fashion anterior to the vitreous (Fig. 4). Some 


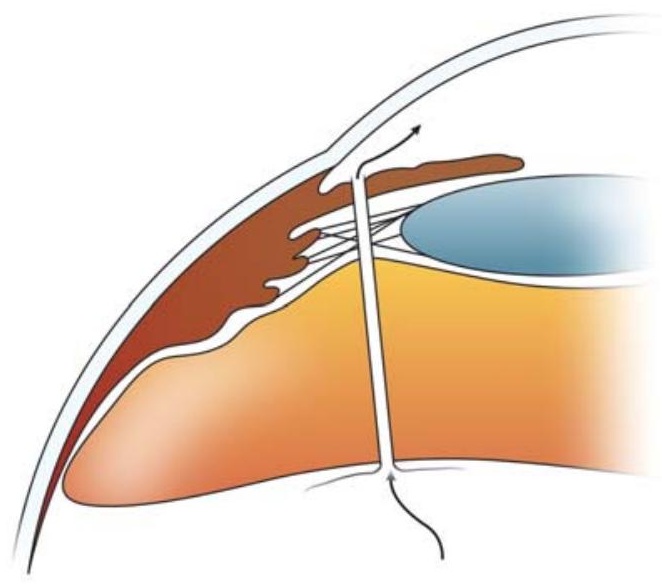

Fig. 3: Schematic depiction of a surgically created route for aqueous flow in the therapy of malignant glaucoma. Multiple techniques may create this route including laser, vitrector and needle based procedures

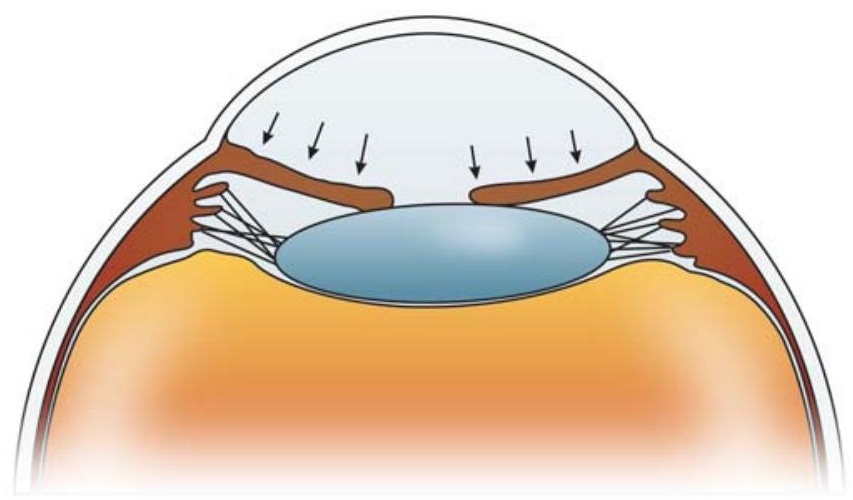

Fig. 4: Schematic depiction of anterior chamber deepening, posterior movement of the vitreous face and re-establishment of the posterior chamber. Generally requires prior creation of space within posterior segment to allow for anterior segment expansion

procedures likely involve components of both of these mechanisms.

\section{Create Conditions for Increased Flow of Fluid through Vitreous Gel}

\section{Laser Procedures}

Disruption of the anterior vitreous using the Q-switched yttrium aluminum garnet (YAG) laser may be effective in alleviating MG. ${ }^{17-21}$ It has been suggested that the mechanism involves puncturing the anterior hyaloid face and providing a new communication between sequestered aqueous and the anterior chamber. ${ }^{17}$ However, evidence that the most anterior vitreous is compositionally distinct from the rest of the vitreous is lacking. Hence, an alternative hypothesis suggests that YAG laser application to the anterior vitreous induces relatively widespread and deeper alterations of the vitreous structure, which are, in turn responsible for improved fluid conductivity of the vitreous gel. $^{22}$

The anterior vitreous may be accessed with the YAG laser either through a large peripheral iridotomy or iridectomy, or through the pupil. Although often successful, the former requires a large peripheral iridotomy or iridectomy and visualization may be poor. The difficulty of this approach is compounded in phakic eyes as one endevours to avoid iatrogenic lens trauma. The alternative trans-pupil approach to YAG laser application has been described in phakic eyes but is generally reserved for pseudophakic eyes. ${ }^{18}$ In pseudophakic eyes, the laser "hyaloidotomy" is carried out with concomitant capsulotomy. ${ }^{19,21}$ Despite this combination approach, unobstructed flow of sequestered aqueous will not occur in some eyes as vitreous and or lens capsule may continue to block the communication.

\section{Incisional Procedures}

Should attempts at establishing a new route for anterior aqueous flow via use of the YAG laser fail, or be deemed inappropriate due to the lens status, incisional interventions may be capable of creating such a communication. Chandler's classical technique was a multiple step procedure in which the first steps served diagnostic purposes. ${ }^{11,23}$ These involved ensuring the patency of the peripheral iridectomy and draining any suprachoroidal fluid. The ensuing steps originally involved inserting a needle between the lens and ciliary body and proceeding to pierce the anterior hyaloid. Because this approach resulted in many lens complications, a pars plana approach to insertion of the needle was subsequently substituted. Following needle insertion, vitreous was aspirated in an attempt to remove a large volume of fluid $(1-1.5 \mathrm{ml})$. This was immediately followed by anterior chamber deepening with balanced saline solution. At the conclusion of the procedure the eye was left hypotonous. ${ }^{11}$

Later techniques have been described which also aim to establish a new route for anterior flow of sequestered aqueous. Using an anterior chamber approach, a needle or vitrector may be introduced through a peripheral iridotomy or iridectomy to mechanically create a new communication through the vitreous. ${ }^{24,25}$ Both procedures have been reported to be safe and effective in pseudophakic eyes and can be performed by anterior segment surgeons.

Finally, pars plana vitrectomy may be required to re-establish normal aqueous flow patterns. ${ }^{26-30}$ However, vitrectomy may fail to alleviate MG if the anterior vitreous is left intact. ${ }^{29,30}$ Hence, in order to ensure anterior vitreous removal and the establishment of a communication with the anterior chamber, some advocate using the vitrector to create a "zonulohyaloidectomy” and peripheral iridectomy as part of the pars plana vitrectomy procedure in pseudophakic eyes. ${ }^{28,29}$ 
Complete and safe removal of anterior vitreous is particularly difficult in phakic patients. As a result, failure to control MG following vitrectomy may be as high as $30-50 \%$ in phakic patients. ${ }^{29,30}$ In response, some have suggested concomitant lensectomy. ${ }^{31}$ However, this of course adds to morbidity. Alternatively, a sequential approach involving first a core vitrectomy followed by phacoemulsification and IOL implantation, and finally completion of the vitrectomy with attention directed at anterior hyaloid removal appears to provide good outcomes. ${ }^{12}$ Finally, endoscopically guided vitrectomy may provide for safer and more complete anterior hyaloid removal in phakic MG. ${ }^{32}$

Historically, lens removal was considered a treatment for MG. The success of this therapy may relate more to disruption of the anterior vitreous than to lens removal as both pseudophakic and aphakic MG are well-described. More recent data show that phacoemulsification, in combination with posterior capsulotomy and anterior vitrectomy, remains a viable option in the management of MG. ${ }^{14}$ Appropriate preparations for dealing with the technical challenges of cataract extraction in an eye with MG, and the associated shallow anterior chamber and vitreous "positive pressure" should be made when considering this option. One approach to these challenges involves vitreous debulking prior to phacoemulsification. This may be carried out via pars plana needle aspiration or via pars plana core vitrectomy.

\section{Restore Physiologic Anatomic Relationships and Re-establish Posterior Chamber}

Attempts to simply reform the anterior chamber with balanced saline or viscoelastic in cases of MG are unlikely to succeed, as the "posterior pressure" is difficult to overcome. In order to allow for the posterior movement of the anteriorly displaced vitreous, space must first be created posteriorly. Hence, aspiration of fluid from the central vitreous cavity, followed by anterior chamber reformation may be successful. This is to a large degree Chandler's technique, suggesting that Chandler's classic approach may function via both mechanisms outlined in this review.

\section{Role of Effusions}

It has long been known that choroidal effusions may induce angle closure in a fashion that may closely mimic MG. ${ }^{33}$ Indeed, Chandler and colleagues described the routine use of posterior sclerotomies in the surgical treatment of presumed MG to rule out the possible association of choroidal effusion. ${ }^{11}$ Clearly there are instances of large effusions, occurring in the absence of hypotony, which result in angle closure. An inflammatory response leading to increased transudation through choroidal vessels has been presumed in many such cases. Examples of etiologies include: Reactions to drugs, human immunodeficiency virus infection, Vogt Koyanagi Harada syndrome, scleritis and pan-retinal photocoagulation. ${ }^{34-38}$ Other etiologies involve poor trans-scleral or vortex vein outflow and include: Nanophthalmos, uveal effusion syndrome and scleral buckling surgery. ${ }^{39-41}$ Clearly in these instances therapy should be directed at the underlying disorder as medical or surgical reduction of the effusion and anterior chamber reformation may relieve the angle closure.

The role of relatively shallow annular ciliary effusions in cases of MG is somewhat controversial. Such effusions have been detected by ultrasound biomicroscopy or B-scan ultrasound in association with cases clinically indistinguishable from MG. ${ }^{42,43}$ Indeed, many of these cases may overlap pathophysiologically with MG and involve a component of aqueous sequestration within or behind the vitreous. ${ }^{5,6}$ However, the extent to which these cases represent a clinically distinct disorder, or simply demonstrate a step in the development or a sequela of MG, is not known with certainty. Nevertheless, drainage of these effusions has successfully resolved anterior chamber shallowing and angle closure in some cases. ${ }^{42,43}$ In contrast, other cases with small ciliary effusions and clinical signs consistent with MG have responded to therapies used in MG without effusion, and without requiring surgical drainage of the supraciliary fluid. ${ }^{5,42,43}$ This suggests that, at least in some cases, small ciliary effusions are associated with the same aqueous retention and sequestration within or behind the vitreous that is involved in MG without effusion.

\section{Other Therapies}

Laser photocoagulation of the ciliary body may also be used in the treatment of MG. Argon laser may be directly applied to the ciliary processes through a peripheral iridectomy. ${ }^{44}$ Alternatively, trans-scleral cyclophotocoagulation using a diode laser has also been described. ${ }^{45-47}$ These therapies have been suggested to achieve their beneficial effects by inducing coagulative shrinkage and posterior rotation of the CB processes. As a consequence, this has been felt to eliminate the abnormal vitreociliary relationship that leads to MG. Alternatively, laser applied to the ciliary body may alter the adjacent vitreous and induce improved fluid conductivity of the vitreous.

\section{SUMMARY}

Malignant glaucoma is a sight-threatening situation. As our understanding of the events involved in this disorder has evolved, so too have our therapies. Careful evaluation of risk factors and appropriate precautions may prevent MG in some cases. Failing that, aggressive cycloplegia in combination with aqueous suppression and hyperosmotic therapy may successfully interrupt the cycle in approximately $50 \%$ of patients over a number of days. Finally, we have a number of laser and 
surgical interventions in our armamentarium that allow for a rational approach to interventional therapy in malignant glaucoma.

\section{REFERENCES}

1. Shaffer RN. The role of vitreous detachment in aphakic and malignant glaucoma. Trans Am Acad Ophthalmol Otolaryngol. 1954;58:217-31.

2. Luntz MH, Rosenblatt M. Malignant glaucoma. Surv Ophthalmol. 1987;32:73-93.

3. Quigley HA, Friedman DS, Congdon NG. Possible mechanisms of primary angle-closure and malignant glaucoma. J Glaucoma. 2003;12:167-80.

4. Epstein DL, Hashimoto JM, Anderson PJ, Grant WM. Experimental perfusions through the anterior and vitreous chambers with possible relationships to malignant glaucoma. Am J Ophthalmol. 1979;88:1078-86.

5. Trope GE, Pavlin CJ, Bau A, Baumal CR, Foster FS. Malignant glaucoma. Clinical and ultrasound biomicroscopic features. Ophthalmology. 1994;101:1030-35.

6. Pavlin CJ, Buys Y, Pathmanathan T, Trope G. Annular peripheral choroidal detachment after glaucoma surgery. Ophthalmology. 1997; 104:1983-84.

7. Quigley HA. Malignant glaucoma and fluid flow rate. Am J Ophthalmol. 1980;89:879-80.

8. Fellman R, Budenz D. Malignant glaucoma. J Glaucoma. 1999;8:149-53.

9. Tello C, Tran HV, Liebmann J, Ritch R. Angle closure: Classification, concepts, and the role of ultrasound biomicroscopy in diagnosis and treatment. Semin Ophthalmol. 2002;17:69-78.

10. Fellman R, Budenz D. Malignant glaucoma. J Glaucoma. 1999;8:149-53.

11. Chandler PA, Simmons RJ, Grant WM. Malignant glaucoma. medical and surgical treatment. Am J Ophthalmol. 1968;66:495502.

12. Sharma A, Sii F, Shah P, Kirkby GR. Vitrectomyphacoemulsification-vitrectomy for the management of aqueous misdirection syndromes in phakic eyes. Ophthalmology. 2006;113:1968-73.

13. Chandler PA, Grant WM. Mydriatic-cycloplegic treatment in malignant glaucoma. Arch Ophthalmol. 1962;68:353-59.

14. Ruben S, Tsai J, Hitchings RA. Malignant glaucoma and its management. Br J Ophthalmol. 1997;81:163-67.

15. Tsai JC, Khaw PT, Hitchings RA. Management of pseudophakic malignant glaucoma. Ophthalmology. 2002;109:820-21.

16. Simmons RJ. Malignant glaucoma. Br J Ophthalmol. 1972;56:263-72.

17. Epstein DL, Steinert RF, Puliafito CA. Neodymium-YAG laser therapy to the anterior hyaloid in aphakic malignant (ciliovitreal block) glaucoma. Am J Ophthalmol. 1984;98:137-43.

18. Brown RH, Lynch MG, Tearse JE, Nunn RD. NeodymiumYAG vitreous surgery for phakic and pseudophakic malignant glaucoma. Arch Ophthalmol. 1986;104:1464-66.
19. Tomey KF, Traverso CE. Neodymium-YAG laser posterior capsulotomy for the treatment of aphakic and pseudophakic pupillary block. Am J Ophthalmol. 1987;104:502-07.

20. Little BC. Treatment of aphakic malignant glaucoma using nd:YAG laser posterior capsulotomy. Br J Ophthalmol. 1994;78:499-501.

21. Little BC, Hitchings RA. Pseudophakic malignant glaucoma: Nd:YAG capsulotomy as a primary treatment. Eye. 1993;7 ( Pt 1):102-04.

22. Teichmann K, Quigley HA. Malignant glaucoma, an enigma solved? J Glaucoma. 2003;12:446-47; author reply 447-48.

23. Chandler PA. A new operation for malignant glaucoma: A preliminary report. Trans Am Ophthalmol Soc. 1964;62:408-24.

24. Francis BA, Wong RM, Minckler DS. Slit-lamp needle revision for aqueous misdirection after trabeculectomy. J Glaucoma. 2002;11:183-88.

25. Lois N, Wong D, Groenewald C. New surgical approach in the management of pseudophakic malignant glaucoma. Ophthalmology. 2001;108:780-83.

26. Weiss H, Shin DH, Kollarits CR. Vitrectomy for malignant (ciliary block) glaucomas. Int Ophthalmol Clin. 1981;21:11319.

27. Momoeda S, Hayashi H, Oshima K. Anterior pars plana vitrectomy for phakic malignant glaucoma. Jpn J Ophthalmol. 1983;27:73-79.

28. Lynch MG, Brown RH, Michels RG, Pollack IP, Stark WJ. Surgical vitrectomy for pseudophakic malignant glaucoma. Am J Ophthalmol. 1986;102:149-53.

29. Byrnes GA, Leen MM, Wong TP, Benson WE. Vitrectomy for ciliary block (malignant) glaucoma. Ophthalmology. 1995;102:1308-11.

30. Harbour JW, Rubsamen PE, Palmberg P. Pars plana vitrectomy in the management of phakic and pseudophakic malignant glaucoma. Arch Ophthalmol. 1996;114:1073-78.

31. Cekic O, Batman C. Pars plana vitrectomy in the treatment of phakic and pseudophakic malignant glaucoma. Arch Ophthalmol. 1998;116:118.

32. Chen SD, Salmon JF, Patel CK. Videoendoscope-guided fluorescein-assisted vitrectomy for phakic malignant glaucoma. Arch Ophthalmol. 2005;123:1419-21.

33. Fourman S. Angle-closure glaucoma complicating ciliochoroidal detachment. Ophthalmology. 1989;96:646-53.

34. Fraunfelder FW, Fraunfelder FT, Keates EU. Topiramateassociated acute, bilateral, secondary angle-closure glaucoma. Ophthalmology. 2004;111:109-11.

35. Ullman S, Wilson RP, Schwartz L. Bilateral angle-closure glaucoma in association with the acquired immune deficiency syndrome. Am J Ophthalmol. 1986;101:419-24.

36. Moorthy RS, Inomata H, Rao NA. Vogt-koyanagi-harada syndrome. Surv Ophthalmol. 1995;39:265-92.

37. Quinlan MP, Hitchings RA. Angle-closure glaucoma secondary to posterior scleritis. Br J Ophthalmol. 1978;62:330-35.

38. Blondeau P, Pavan PR, Phelps CD. Acute pressure elevation following panretinal photocoagulation. Arch Ophthalmol. 1981;99:1239-41. 
39. Brockhurst RJ. Nanophthalmos with uveal effusion. A new clinical entity. Arch Ophthalmol. 1975;93:1989-99.

40. Gass JD, Jallow S. Idiopathic serous detachment of the choroid, ciliary body, and retina (uveal effusion syndrome). Ophthalmology. 1982;89:1018-32.

41. Regan CD, Schepens CL, Okamura ID, Brockhurst RJ, McMeel JW. The scleral buckling procedures. VI. further notes on silicone in primary operations. Arch Ophthalmol. 1962;68:313-28.

42. Dugel PU, Heuer DK, Thach AB, et al. Annular peripheral choroidal detachment simulating aqueous misdirection after glaucoma surgery. Ophthalmology. 1997;104:439-44.

43. Liebmann JM, Weinreb RN, Ritch R. Angle-closure glaucoma associated with occult annular ciliary body detachment. Arch Ophthalmol. 1998;116:731-35.

44. Herschler J. Laser shrinkage of the ciliary processes. A treatment for malignant (ciliary block) glaucoma. Ophthalmology. 1980;87:1155-59.

45. Carassa RG, Bettin P, Fiori M, Brancato R. Treatment of malignant glaucoma with contact transscleral cyclophotocoagulation. Arch Ophthalmol. 1999;117:688-90.

46. Sengupta R, Austin M, Morgan J. Treatment of aqueous misdirection by trans-scleral diode laser photocoagulation. Eye. 2000;14 Pt 5:808-10.
47. Muqit MM, Menage MJ. Malignant glaucoma after phacoemulsification: Treatment with diode laser cyclophotocoagulation. J Cataract Refract Surg. 2007;33:13032.

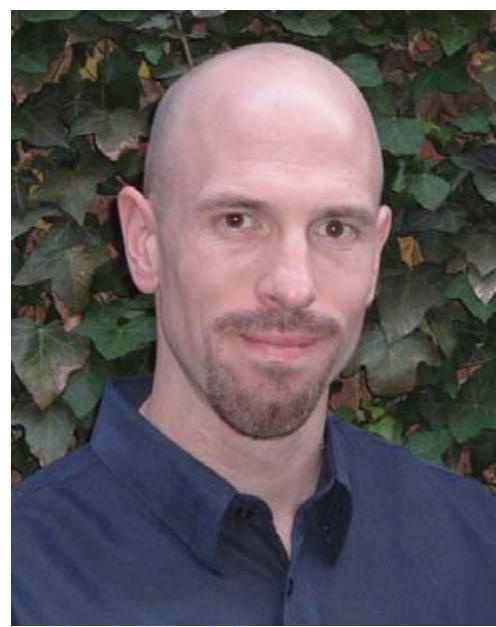

Robert J Campbell

(rob.campbell@queensu.ca)

"I would rather be ash than dust.

I would rather my spark should burn out in a brilliant blaze,

Than it should be stifled in dry rot.

I would rather be a superb meteor,

Every atom of me in magnificent glow,

Than a sleepy and permanent planet.

Man's chief purpose is to live, not exist.

I shall not waste my days trying to prolong them.

I shall use my time.” 